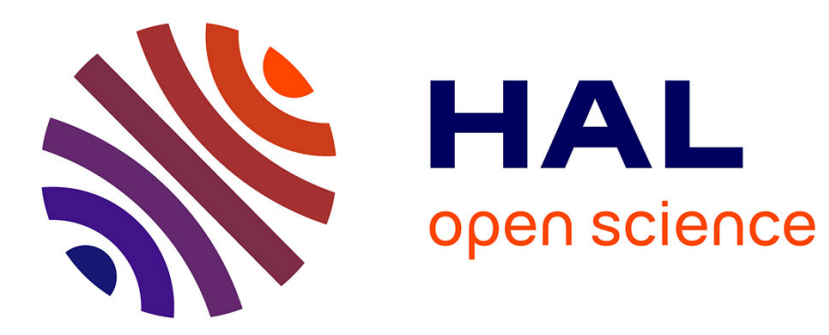

\title{
Comportement dynamique des lasers semiconducteurs en régime de modulation directe
}

\author{
E. Hemery, J.-M. Lourtioz
}

\section{To cite this version:}

E. Hemery, J.-M. Lourtioz. Comportement dynamique des lasers semiconducteurs en régime de modulation directe. Revue de Physique Appliquée, 1987, 22 (11), pp.1571-1580. 10.1051/rphysap:0198700220110157100 . jpa-00245709

HAL Id: jpa-00245709

https://hal.science/jpa-00245709

Submitted on 1 Jan 1987

HAL is a multi-disciplinary open access archive for the deposit and dissemination of scientific research documents, whether they are published or not. The documents may come from teaching and research institutions in France or abroad, or from public or private research centers.
L'archive ouverte pluridisciplinaire HAL, est destinée au dépôt et à la diffusion de documents scientifiques de niveau recherche, publiés ou non, émanant des établissements d'enseignement et de recherche français ou étrangers, des laboratoires publics ou privés. 


\title{
Comportement dynamique des lasers semiconducteurs en régime de modulation directe
}

\author{
E. Hemery et J.-M. Lourtioz \\ Institut d'Electronique Fondamentale, Laboratoire associé au CNRS, UA 022 Université Paris-Sud, Bâtiment \\ 220, 91405 Orsay Cedex, France
}

(Reçu le 12 mars 1987, accepté le 14 mai 1987)

\begin{abstract}
Résumé. - Une étude détaillée des comportements dynamiques des lasers à semiconducteurs modulés en courant est présentée. La non-linéarité du gain est prise en compte dans les équations laser. Une méthode de résolution numérique performante permet de donner les résultats sous forme de diagrammes de bifurcation dans l'espace 3d des paramètres de modulation. Il est montré que la possibilité de comportements instables est directement liée au facteur d'amortissement du laser. En ce qui concerne les lasers InGaAsP dont l'amortissement est généralement élevé, il est essentiellement prédit un comportement périodique simple. Des oscillations de relaxation peuvent néanmoins se manifester à basse fréquence de modulation. En ce qui concerne les lasers AsGa à plus faible amortissement, des doublements de période sont possibles, soit à basse ou à haute fréquence de modulation. En revanche, un comportement chaotique semble exclu pour des valeurs réalistes de paramètres-laser.
\end{abstract}

\begin{abstract}
A detailed study of the dynamic response of directly modulated semiconductor lasers is presented. The non-linearity of the gain is included in the laser equations. A performing numerical simulation method allows us to present the results in the form of bifurcation diagrams in the $3 \mathrm{~d}$ space of modulation parameters. It is shown that the occurrence of unstable behaviours is directly related to the laser damping coefficient. Concerning InGaAsP lasers which generally exhibit strong damping, a simple periodic response is mostly predicted. Nevertheless, oscillation relaxations may occur within a single periode at low modulation frequencies. For AsGa lasers which exhibit a weakest damping period doubling may occur either at low or high modulation frequencies. In contrast, a chaotic behaviour seems to be excluded for realistic values of the laser parameters.
\end{abstract}

\section{Introduction.}

$\mathrm{Au}$ cours des dernières années, de nombreux travaux ont été consacrés à l'optimisation des diodes-laser modulées en courant. Ces dispositifs présentent en effet des applications particulièrement importantes dans le domaine des communications optiques $[1,2]$. D'autres domaines d'application sont d'ailleurs envisageables; l'utilisation récente de diodes laser InGaAsP modulées en HF pour l'échantillonnage électro-optique de circuits microélectroniques AsGa en est un exemple [3].

Dans la plupart des travaux d'optimisation, les performances en fréquence des dispositifs ont été caractérisées en régime de faible signal $[4,11]$. Les résultats de réponse en fréquence publiés dans la référence [12] sont parmi les seuls où des taux de modulation de $\sim 65 \%$ ont été envisagés. Or, si l'approche linéarisée du faible signal reste a priori applicable jusqu'à des taux de modulation modérés, elle devient en revanche illégitime aux fortes modulations. Dans l'approximation monomode, on peut considérer le laser à semiconducteur modulé en courant comme un système non linéaire à trois degrés de libertés, lesquels sont respectivement la densité de porteurs, la densité de photons et le courant de modulation ; un tel système peut présenter des comportements à tendance chaotique [13a]. Un comportement de type doublement de période a ainsi été observé expérimentalement pour des diodes AsGa et des taux de modulation voisins de l'unité [14]. Ce résultat a été apparemment confirmé par une première étude de simulation numérique [15], laquelle prédisait également la possibilité de comportement chaotique et de comportement périodique avec apparition de pics multiples pour chaque 
période de modulation. Une seconde étude théorique [16] indiquait qu'en revanche les comportements de type chaotique ou doublement de période ne devaient pas être observés pour les diodes InGaAsP à $1,3 \mu \mathrm{m}$, ceci dû à une non-linéarité accrue du gain laser pour ce type de matériau [17]. Notons cependant que, dans cette seconde étude, il n'est fait aucunement mention des comportements périodiques anormaux avec pics multiples.

Dans cet article, nous présentons une étude topologique détaillée des comportements-laser dans l'espace associé aux paramètres de modulation. Des dispositifs AsGa et InGaAsP sont considérés simultanément. A la différence de la référence [15], la non-linéarité du gain est prise en compte dans les équations-laser. A la différence des références [15] et [16], les valeurs des paramètres-laser sont choisies de manière à correspondre à des dispositifs performants en fréquence. Nous montrons également comment la possibilité de tel ou tel comportementlaser peut être diagnostiquée à partir des courbes de réponse en faible signal. De ce fait, l'exposé qui suit se présente en trois parties. Après avoir rappelé dans une première partie les équations de base du laser et les approximations commises, nous présentons dans la seconde partie les lois d'évolution de la répónse en faible signal. Les résultats de simulation numérique en fort signal sont présentés dans la troisième partie, laquelle est suivie d'une conclusion.

\section{Equations du laser.}

Suivant l'approximation monomode, les équations de base du laser à semiconducteur modulé en courant s'écrivent :

$$
\begin{aligned}
\mathrm{d} N / \mathrm{d} t & =I / e V-N / \tau_{\mathrm{n}}-A\left(N-N_{0}\right) S(1-\varepsilon S) \\
\mathrm{d} S / \mathrm{d} t & =-S / \tau_{\mathrm{p}}+\Gamma \beta N / \tau_{\mathrm{n}}+\Gamma A\left(N-N_{0}\right) S(1-\varepsilon S) \\
I & =I_{\mathrm{p}}+I_{\mathrm{m}} \sin \omega t
\end{aligned}
$$

L'équation (1a) décrit l'évolution temporelle de la densité de porteurs. Dans cette équation, les trois termes représentent respectivement : le taux d'injection de porteurs dans la zone active, le taux de recombinaison spontanée et le taux de recombinaison par émission stimulée. L'équation (1b) décrit l'évolution temporelle de la densité de photons. Dans cette équation les trois termes représentent respectivement : les pertes de cavité incluant à la fois le couplage et l'absorption dans la zone active, la contribution de l'émission spontanée au mode de cavité et l'émission stimulée. La définition de chacun des paramètres-laser intervenant dans les équations (1a) et (1b) est rappelée dans le tableau I. La modulation en courant est ici supposée sinusoïdale
(Eq. (1c)). $I_{\mathrm{p}}$ est le courant de polarisation et $I_{\mathrm{m}}$, l'amplitude de la modulation.

En toute généralité, le coefficient de gain $A$, le paramètre $\varepsilon$ qui décrit la non-linéarité du gain («spectral hole burning ») et le temps de recombinaison spontanée $\tau_{\mathrm{n}}$ dépendent de la densité de porteurs. Les variations de $A$ et $\varepsilon$ en fonction de $N$ peuvent être déduites de la référence [17]. D'autre part, une expression communément admise pour $\left(N / \tau_{0}\right)$ est de la forme $\alpha N+B N^{2}+C N^{3}$ où $B N^{2}$ est associé au processus de recombinaison radiative tandis que $\alpha N$ et $C N^{3}$ sont associés à des processus de recombinaison non radiative [18-20]. Dans les études de simulation, nous avons pris $\tau_{\mathrm{n}}, A$ et $\varepsilon$ constants. Ceci revient à supposer que la

\begin{tabular}{|c|c|c|c|}
\hline Paramètres laser & Laser I & Laser II & Laser III \\
\hline $\begin{array}{ll}\varepsilon & : \text { Facteur de saturation de gain. } \\
N_{0} & : \text { Densité de porteurs à la transparence. } \\
A & : \text { Constante de gain. } \\
\Gamma & : \text { Facteur de confinement. } \\
\tau_{\mathrm{n}} & : \text { Durée de vie des porteurs. } \\
\tau_{\mathrm{p}} & : \text { Durée de vie des photons. } \\
\beta & : \text { Taux d'émission spontanée dans le mode. } \\
V & : \text { Volume de la couche active. }\end{array}$ & $\begin{array}{c}2,0 \mathrm{E}-17 \mathrm{~cm}^{+3} \\
2 \mathrm{E}+18 \mathrm{~cm}^{-3} \\
2,0 \mathrm{E}-6 \mathrm{~cm}^{+3} / \mathrm{s} \\
0,5 \\
0,7 \mathrm{E}-9 \mathrm{~s} \\
0,42 \mathrm{E}-12 \mathrm{~s} \\
4,0 \mathrm{E}-4 \\
100 \mu \mathrm{m} \\
\times 0,25 \mu \mathrm{m} \\
\times 1,5 \mu \mathrm{m} \\
0,45\end{array}$ & $\begin{array}{c}1,5 \mathrm{E}-17 \mathrm{~cm}^{+3} \\
1 \mathrm{E}+18 \mathrm{~cm}^{-3} \\
2,5 \mathrm{E}-6 \mathrm{~cm}^{+3} / \mathrm{s} \\
0,5 \\
2,2 \mathrm{E}-9 \mathrm{~s} \\
1,2 \mathrm{E}-12 \mathrm{~s} \\
3,4 \mathrm{E}-4 \\
120 \mu \mathrm{m} \\
\times 0,15 \mu \mathrm{m} \\
\times 4,4 \mu \mathrm{m} \\
0,35\end{array}$ & $\begin{array}{c}0,3 \mathrm{E}-17 \mathrm{~cm}^{+3} \\
1,5+18 \mathrm{~cm}^{-3} \\
2,5 \mathrm{E}-7 \mathrm{~cm}^{+3} / \mathrm{s} \\
0,8 \\
3 \mathrm{E}-9 \mathrm{~s} \\
6 \mathrm{E}-12 \mathrm{~s} \\
5 \mathrm{E}-5 \\
2,5 \mathrm{E}-10 \mathrm{~cm}^{+3}\end{array}$ \\
\hline
\end{tabular}

\section{Tableau I. - Définition des paramètres-laser et valeurs choisies pour la simulation numérique.}

[Laser parameters-definitions and values used for the numerical simulation.] 
densité de porteurs évolue peu autour de la valeur stationnaire, $N_{\mathrm{p}}$, correspondant au point de polarisation. Cette approximation a été justifiée $a$ posteriori. Les résultats de simulation numérique ont en effet montré que la variation relative de la densité de porteurs n'excédait jamais $\sim 10^{-3}$, y compris dans le cas de fortes modulations, sauf si la fréquence de modulation était inférieure à $\left(1 / 2 \pi \tau_{\mathrm{n}}\right)\left({ }^{1}\right)$. Il est par contre évident que l'approximation précédente est moins justifiable dans le cas où l'on considère la réponse à une impulsion de courant avec $I_{\mathrm{p}}=0$ au repos.

Une autre approximation commise dans les équations du laser concerne l'omission des effets de diffusion de porteurs dans la couche active [21-24] et de l'absorption non linéaire à deux photons $[25,26]$. L'importance des effets de diffusion peut être caractérisée par le rapport $h=\left(2 \pi L_{\mathrm{D}} / W\right)^{2}$, où $L_{\mathrm{D}}$ est la longueur de diffusion et $W$ la largeur du ruban. Une résolution approchée des équations-laser dans le cas où les effets de diffusion sont pris en compte [22] montre que l'on peut se ramener aux équations (1a) et (1b), à condition d'intégrer le terme $\tau_{\mathrm{n}} A / 2(1+h)$ dans $\varepsilon$. De même, la prise en compte de l'absorption à deux photons se traduit par un terme supplémentaire de la forme $\gamma S^{2}$ dans l'équation (1b), ce qui revient en première approximation à accroître la valeur de $\varepsilon$. On peut donc admettre que le terme $\varepsilon$ dans l'équation (1b) décrit non seulement la non-linéarité du gain laser, mais aussi les effets de diffusion de porteurs et l'absorption à deux photons.

Les valeurs des paramètres-laser choisies pour la simulation numérique sont données dans les colonnes 2, 3, 4 du tableau I. Les valeurs de la colonne 2 correspondent approximativement à un laser InGaAsP à ruban enterré, court et fortement dopé [10]. La faible longueur du ruban actif se traduit par une faible valeur de $\tau_{\mathrm{p}}$ tandis que le fort dopage se traduit par un coefficient de gain élevé et une durée de vie des porteurs relativement courte. Les paramètres $\beta$ et $\varepsilon$ ont été ajustés de manière à reproduire les courbes de réponse en faible signal rapportées dans la référence [10]. Notons au passage que la valeur de $\varepsilon$ trouvée est proche de celles estimées pour d'autres lasers InGaAsP [6].

Les valeurs des paramètres rapportées dans la colonne 3 correspondent à un laser $\mathrm{AsGa}$ à ruban enterré sur substrat semi-isolant [7]. Là encore, les paramètres $\beta$ et $\varepsilon$ ont été ajustés de manière à

( $\left.{ }^{1}\right)$ D'après l'équation (1a), en faisant $S=0$, la fréquence $f_{\mathrm{c}}=1 / 2 \pi \tau_{\mathrm{n}}$ apparaît comme la fréquence de coupure d'un filtre passe-bas. Si la fréquence de modulation est inférieure à $f_{\mathrm{c}}$ et si le taux de modulation est suffisamment élevé de telle sorte que l'on passe en dessous du seuil-laser $(S \rightarrow 0)$, alors $N$ pourra varier de $N_{\mathrm{p}}$ à $\sim 0$. approcher les courbes de réponse en faible signal données par les auteurs.

Les valeurs des paramètres-laser rapportées dans la colonne 4 , excepté celle de $\varepsilon$, sont identiques à celles utilisées dans les travaux les plus récents de simulation numérique en fort signal $[15,16]$. elles correspondent $a$ priori à un laser long et à gain modéré. A la différence de la référence [15], $\varepsilon$ a été pris différent de zéro. La valeur choisie est la valeur théorique minimum envisageable pour un laser AsGa [17], abstraction faite des effets de diffusion ou d'absorption à deux photons.

\section{Solutions stationnaires et réponse en faible signal.}

3.1 Solutions STATIONNAIRES. - En régime stationnaire $\left(\mathrm{d} N / \mathrm{d} t=\mathrm{d} S / \mathrm{d} t=I_{\mathrm{m}}=0\right)$, on montre facilement par une combinaison des équations (1a) et (1b) que les densités de porteurs et de photons, $N$ et $S$, sont reliées par:

$$
N=\frac{\tau_{\mathrm{n}}}{(1-\beta)}\left[\frac{I_{\mathrm{p}}}{e V}-\frac{S}{\Gamma \tau_{\mathrm{p}}}\right] .
$$

L'utilisation de cette relation dans l'équation (1b) conduit à une équation du troisième degré en $S$, dont la racine réelle positive est la solution du régime stationnaire. En utilisant les quantités usuelles $N_{\mathrm{s}}=N_{0}+1 / A \Gamma \tau_{\mathrm{p}}$ et $I_{\mathrm{s}}=\left(\mathrm{eV} / \tau_{\mathrm{n}}\right) N_{\mathrm{s}}$, qui sont respectivement la densité de porteurs au seuil-laser et le courant de seuil, l'équation du troisième degré se met sous la forme :

$$
\begin{aligned}
\varepsilon S^{3}- & {\left[1+\varepsilon\left(\frac{\Gamma \tau_{\mathrm{p}}}{e V}\left(I_{\mathrm{p}}-I_{\mathrm{s}}\right)-\frac{1}{A \tau_{\mathrm{n}}}\right)\right] S^{2}+} \\
& +\frac{\Gamma \tau_{\mathrm{p}}}{e V}\left(I_{\mathrm{p}}-I_{\mathrm{s}}+\frac{\beta e V N_{0}}{\tau_{\mathrm{n}}}\right) S+\frac{\Gamma \tau_{\mathrm{p}} \beta I_{\mathrm{p}}}{A \tau_{\mathrm{n}} e V}=0 .
\end{aligned}
$$

L'expression algébrique exacte de la racine réelle positive de cette équation a été utilisée dans la programmation numérique, mais son intérêt est limité de par sa complexité. Des expressions simplifiées peuvent en revanche être établies après examen des différents coefficients de l'équation (2b). Notons d'abord que le coefficient du terme en $S$ dans l'équation (2b) se réduit toujours à $\Gamma \tau_{\mathrm{p}}\left(I_{\mathrm{p}}-I_{\mathrm{s}}\right) / \mathrm{eV}$ dès que $\left|I_{\mathrm{p}}-I_{\mathrm{s}}\right| \geqslant 10 \mu \mathrm{A}$.

En dessous du seuil $\left(I_{\mathrm{p}}<I_{\mathrm{s}}, \varepsilon S \ll 1\right)$, l'équation $(2 b)$ se ramène à une équation du second degré en faisant $\varepsilon=0$ :

$$
S^{2}-\frac{\Gamma \tau_{\mathrm{p}}}{e V}\left(I_{\mathrm{p}}-I_{\mathrm{s}}\right) . S-\frac{\Gamma \tau_{\mathrm{p}} \beta I_{\mathrm{p}}}{e V A \tau_{\mathrm{n}}}=0 .
$$

On montre facilement que la solution $S_{\mathrm{p}}$ est bien approximée par :

$$
S_{\mathrm{p}} \approx\left(\beta / A \tau_{\mathrm{n}}\right) \cdot I_{\mathrm{p}} /\left(I_{\mathrm{s}}-I_{\mathrm{p}}\right)
$$


ce qui, d'après la relation $(2 \mathrm{a})$, conduit à :

$$
N_{\mathrm{p}} \approx\left(\tau_{\mathrm{n}} / e V\right) \cdot I_{\mathrm{p}}
$$

Au-dessus du seuil $\left(I_{\mathrm{p}}>I_{\mathrm{s}}\right)$, on peut supposer que la contribution due à l'émission spontanée est négligeable, ce qui revient à faire $\beta=0$ dans l'équation (2b). On se ramène alors à nouveau à une équation du second degré :

$$
\begin{array}{r}
\varepsilon S^{2}-\left[1+\varepsilon\left(\frac{\Gamma \tau_{\mathrm{p}}}{e V}\left(I_{\mathrm{p}}-I_{\mathrm{s}}\right)-\frac{1}{A \tau_{\mathrm{n}}}\right)\right] S+ \\
+\frac{\Gamma \tau_{\mathrm{p}}}{e V}\left(I_{\mathrm{p}}-I_{\mathrm{s}}\right)=0
\end{array}
$$

dont la solution approchée est :

$$
\begin{aligned}
S_{\mathrm{p}} \approx\left(\Gamma \tau_{\mathrm{p}} / e V\right) \cdot\left(I_{\mathrm{p}}\right. & \left.-I_{\mathrm{s}}\right)\left(1-\varepsilon / A \tau_{\mathrm{n}}\right) \approx \\
& \approx\left(\Gamma \tau_{\mathrm{p}} / e V\right) \cdot\left(I_{\mathrm{p}}-I_{\mathrm{s}}\right)
\end{aligned}
$$

sachant que $\varepsilon S$ reste malgré tout inférieur à l'unité. En utilisant la relation (2a), la densité de porteur en régime stationnaire s'écrit maintenant:

$$
N_{\mathrm{p}} \approx\left(\tau_{\mathrm{n}} / e V\right) \cdot\left(I_{\mathrm{s}}+\left(I_{\mathrm{p}}-I_{\mathrm{s}}\right) \varepsilon / A \tau_{\mathrm{n}}\right) \approx N_{\mathrm{s}} .
$$

La validité des relations (3 ou 4) est directement illustrée à partir des caractéristiques puissance laser/courant d'injection rapportées dans les encarts des figures $1 \mathrm{a}$ et $1 \mathrm{~b}$, respectivement. La courbe de la figure 1a correspond au laser InGaAsP (colonne 2, Tab. I). Celle de la figure $1 \mathrm{~b}$ correspond au laser AsGa performant (colonne 3, tab. I). La puissance couplée par chacun des miroirs-laser est reliée à la densité de photons par :

$$
P=(1 / 2) \cdot S_{\mathrm{p}} \cdot h \nu \cdot \eta \cdot V / \tau_{\mathrm{p}}
$$

où $\eta$ est le rendement quantique du laser.

Les deux courbes ont été calculées en utilisant l'expression exacte de $S_{\mathrm{p}}$. L'évolution linéaire de $P$ et donc de $S$, avec $I_{\mathrm{p}}$ (relation (4a)) apparaît ainsi clairement dans chaque cas pourvu que $I_{\mathrm{p}} / I_{\mathrm{s}}$ excède l'unité de quelques pourcents. Notons que cette évolution est effectivement observée expérimentalement (voir Réf. [4], par exemple) à condition que le courant de polarisation ne soit pas trop élevé. A fort courant, différents effets (effets thermiques, «sauts » de mode, dégradation des facettesmiroir...) peuvent entraîner une non-linéarité de la caractéristique $P\left(I_{\mathrm{p}}\right)$.

Dans ce qui suit, nous supposerons toujours la diode-laser polarisée au-delà du seuil et les relations $(4 \mathrm{a}, \mathrm{b})$ applicables. $\left(I_{\mathrm{p}} / I_{\mathrm{s}}\right)$ sera défini comme le taux de polarisation avec des valeurs variant de $\sim 1,05$ à $\sim 3$. De même, le rapport $I_{\mathrm{m}} /\left(I_{\mathrm{p}}-I_{\mathrm{s}}\right)$ sera défini comme le taux de modulation.

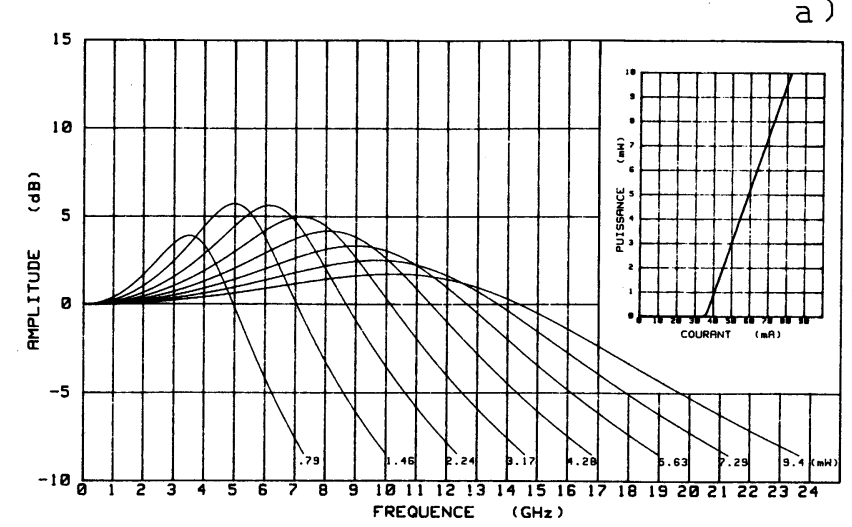

b)

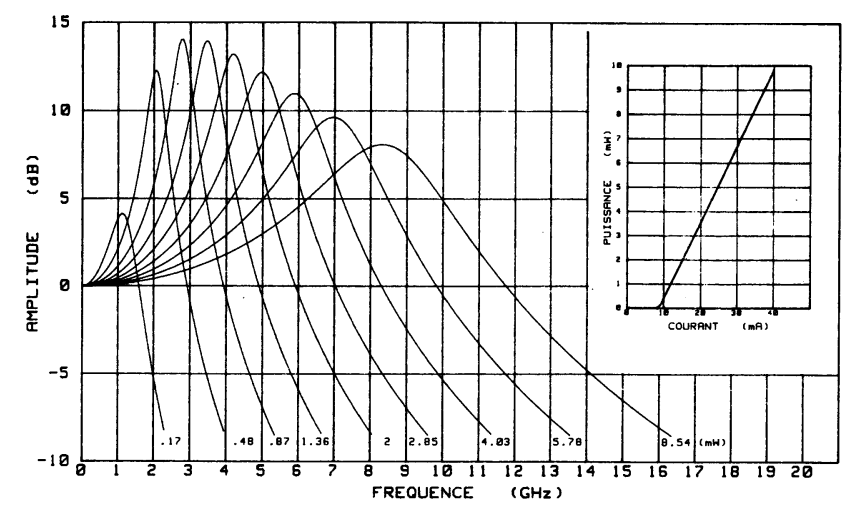

Fig. 1. - Courbes calculées de la réponse en faible signal pour deux des lasers envisagés dans la simulation numérique. Le réseau de courbes de la figure 1a correspond au laser InGaAsP (Laser I, tableau I). Celui de la figure 1b correspond au laser AsGa (Laser II, tableau I). L'amplitude de la réponse est exprimée en décibels. La puissance du signal optique continu est le paramètre de référence pour chacune des courbes. La relation entre puissance optique et courant de polarisation est indiquée par les courbes d'évolution rapportées dans les encarts des figures $1 \mathrm{a}$ et $1 \mathrm{~b}$, respectivement.

[Calculated small-signal frequency responses for two of the three lasers considered in table I. The series of curves of figure $1 \mathrm{a}$ correspond to the InGaAsP laser diode (Laser I, table I). Those of figure $1 \mathrm{~b}$ correspond to the AsGa laser diode (Laser II, table I). The response amplitude is in decibel units. The $\mathrm{CW}$ optical power is the reference parameter for each curve. The relation between optical power and bias current is indicated by the evolution curves reported in the inserts of figure $1 \mathrm{a}$ and $1 \mathrm{~b}$, respectively.]

3.2 RÉPONSE EN FAIBLE SIGNAL. - La réponse en faible signal $\left(I_{\mathrm{m}}=i\right)$ s'obtient par une linéarisation des équations $(1 \mathrm{a}, 1 \mathrm{~b})$ au voisinage du point de polarisation $\left(S=S_{\mathrm{p}}+s\right.$ et $\left.N=N_{\mathrm{p}}+n\right)$. Le comportement dynamique du laser à semiconduteur étant régi par deux équations différentielles, la fonction de transfert $F(j \omega)=s(j \omega) / i(j \omega)$ s'identifie à celle d'un filtre du second ordre. La réponse en fréquence 
du laser, normalisée à la réponse à fréquence nulle, peut donc s'écrire sous la forme :

$$
\frac{F(j \omega)}{F(0)}=\frac{\omega_{0}^{2}}{(j \omega)^{2}+2 \xi \omega_{0}(j \omega)+\omega_{0}^{2}}
$$

où $f_{0}=\omega_{0} / 2 \pi$ est la fréquence propre du filtre équivalent et $\xi$, le facteur d'amortissement.

De façon générale, un tel système présentera une résonance si $\xi<\sqrt{2} / 2$. La fréquence de résonance, $f_{\mathrm{R}}$ sera alors égale à $f_{0} \times \sqrt{1-2 \xi^{2}}$ et l'amplitude $\mathrm{du}$ maximum sera donnée par $\left[2 \xi \sqrt{1-\xi^{2}}\right]^{-1} \sim 1 / 2 \xi$. Considérant par ailleurs la réponse transitoire du système, il est également connu qu'elle présentera des oscillations de relaxation à la fréquence $\sim f_{\mathrm{R}}$ et dont la durée (temps d'amortissement) sera de l'ordre de $1 / \xi \omega_{0}$.

La réponse en fréquence d'un laser à semiconducteur a été analysée de façon détaillée dans la littérature $[2,5,6,9]$. Aussi, nous nous bornerons à indiquer les résultats essentiels :

i) Supposant $A S_{\mathrm{p}}>\beta / \tau_{\mathrm{n}}$, ce qui est largement vérifié pour des taux de polarisation supérieurs à 1,01 , la résolution des équations linéarisées du laser conduit aux relations :

$$
\begin{aligned}
\omega_{0}^{2} & =\frac{A S_{\mathrm{p}}\left(1-\varepsilon S_{\mathrm{p}}\right)}{\tau_{\mathrm{p}}}+\frac{\beta+\varepsilon S_{\mathrm{p}}}{\tau_{\mathrm{p}} \tau_{\mathrm{n}}}+\frac{\beta^{\prime}}{\tau_{\mathrm{n}} S_{\mathrm{p}}} \\
2 \xi \omega_{0} & =\frac{\beta^{\prime}}{S_{\mathrm{p}}}+\left(A+\frac{\varepsilon}{\tau_{\mathrm{p}}}\right) S_{\mathrm{p}}+\frac{1}{\tau_{\mathrm{n}}} \\
\beta^{\prime} & =\beta \Gamma I_{\mathrm{s}} / e V .
\end{aligned}
$$

ii) Pour les taux de polarisation envisagés $(\geqslant 1,05)$, le premier terme de l'équation (7a) est toujours dominant. Sachant par ailleurs que $\varepsilon S_{\mathrm{p}}$ reste aussi largement inférieur à l'unité, la fréquence propre $f_{0}$ s'identifie alors à :

$$
f_{0}^{\prime}=\frac{1}{2 \pi} \sqrt{\frac{A S_{\mathrm{p}}}{\tau_{\mathrm{p}}}} .
$$

Tant que l'amortissement restera modéré, la fréquence de résonance $f_{\mathrm{R}}$ s'identifiera aussi à $f_{0}^{\prime}$ et sera donc proportionnelle à la racine carrée de la puissance optique (relation (5)) ou encore à la racine carrée de $\left(I_{\mathrm{p}}-I_{\mathrm{s}}\right)$ (relation (4a)). Vu en terme de performance en fréquence, la grandeur caractéristique généralement admise est la fréquence de coupure à $-3 \mathrm{~dB}$. Celle-ci étant proportionnelle à $f_{0}$, un laser performant sera donc un laser court, à fort gain, à fort couplage et susceptible d'être polarisé à des taux de polarisation élevés.

iii) Dans la relation (7b), le premier et le second termes sont les termes dominants. Le premier l'emporte aux faibles courants de polarisation alors que le second l'emporte aux forts courants. En revanche, le taux de recombinaison des porteurs influe peu sur le facteur d'amortissement. Etant donné le rôle capital de l'amortissement sur le comportement dynamique du laser en fort signal, il est donc permis d'avancer qu'une prise en compte plus détaillée des mécanismes de recombinaison des porteurs (voir partie 2) ne doit pas modifier de façon significative les résultats présentés dans la partie 4 .

iv) Il existe une puissance optique (c'est-à-dire un courant de polarisation) pour laquelle le facteur d'amortissement est minimum. La valeur de $\xi_{\text {min }}$ peut être obtenue avec une bonne précision en négligeant $1 / \tau_{\mathrm{n}}$ dans la relation (7b) ainsi que $A$ devant $\varepsilon / \tau_{\mathrm{p}}$ et en supposant la fréquence propre donnée par la relation (8). L'amplitude optimale de la réponse en faible signal s'écrit alors :

$$
\left|\frac{F(j \omega)}{F(0)}\right|_{\mathrm{opt}} \simeq \frac{1}{2 \xi_{\min }} \simeq \frac{3}{4}\left[\frac{A^{2} \tau_{\mathrm{p}}}{3 \beta^{\prime} \varepsilon^{3}}\right]^{1 / 4} .
$$

La fréquence à laquelle se produit cet optimum est donnée par :

$$
f_{\mathrm{opt}} \simeq \frac{1}{2 \pi}\left[\frac{3 A^{2} \beta^{\prime}}{\tau_{\mathrm{p}} \varepsilon}\right]^{1 / 4} .
$$

Comme le montre la relation (9a), le terme $\varepsilon$ agit de façon déterminante sur l'amplitude optimale.

Certains des résultats qui viennent d'être évoqués ii) et iv) sont directement illustrés à partir des réseaux de courbes des figures $1 \mathrm{a}$ et $1 \mathrm{~b}$. Chacune des courbes représente l'évolution de l'amplitude normalisée de la réponse en fréquence calculée pour un niveau de puissance optique donné. Le calcul est effectué en utilisant les relations $(5,6,7 \mathrm{a}, 7 \mathrm{~b}, 7 \mathrm{c})$ et l'expression exacte de $S_{\mathrm{p}}$. Le réseau de courbes de la figure 1a correspond au laser InGaAsP (colonne 2 du Tab. I) alors que celui de la figure $1 \mathrm{~b}$ correspond au laser AsGa dont les paramètres sont rapportés dans la colonne 3 du tableau I. On peut vérifier que les courbes reproduisent fidèlement les courbes expérimentales rapportées dans les références [4] et [10], respectivement. Les faibles écarts d'amplitude observés entre théorie et expérience $(\leqslant 2 \mathrm{~dB})$ s'expliquent aisément à partir des effets capacitifs parasites (« RC roll-off») présents dans le circuit électrique expérimental. Notons enfin que les amplitudes maximales obtenues $(\sim 6 \mathrm{~dB}$ et $\sim 12 \mathrm{~dB}$ respectivement) sont typiques des lasers InGaAsP et AsGa présentant de bonnes performances en fréquence [2, $6,10,12,27]$.

\section{Réponse en fort signal.}

4.1 RÉSUltatS DE SIMULATION NUMÉRIQUE. - La résolution numérique complète des équations (1a, $1 b, 1 c, 5)$ dans le cas de fortes modulations a été effectuée par une méthode de type MERSON à pas variable et contrôle d'erreur, qui est en fait une 
méthode de type RUNGE-KUTTA modifiée [28]. La topologie détaillée des comportements-laser dans l'espace $3 \mathrm{~d}$ associé aux paramètres de modulation est obtenue comme suit. Le taux de polarisation est varié de 1,1 à 2,1 par pas de 0,2 . Pour chaque taux de polarisation, l'évolution temporelle de la puissance-laser (ou de la densité de porteurs) et calculée sur 30 périodes de modulation et pour $20 \times 20$ couples de valeurs des paramètres : fréquence de modulation et taux de modulation. La fréquence de modulation est variée de quelques centaines de $\mathrm{MHz}$ à $\sim 2,5 f_{\mathrm{R}}$, où $f_{\mathrm{R}}$ est la fréquence de résonance calculée en faible signal (voir 3.2). Le taux de modulation est varié de 0,1 à 2,1 . L'évolution temporelle de la puissance-laser est analysée à partir de la dixième période de modulation, durée jugée suffisamment longue pour que le régime permanent soit établi. Deux types de comptage sont alors effectués : 1) On compte le nombre de périodes du courant de modulation contenues dans une période du signal optique (= détection du «doublement de période » et des comportements-types menant au chaos). 2) On compte le nombre de maxima du signal optique par période du signal optique (= détection des régimes à pics multiples). Si la période du signal optique est supérieure à 20 périodes de modulation (nombre de périodes utilisé pour le comptage), le comportement est déclaré a priori de type chaotique. En réalité, ceci n'a jamais été observé pour les valeurs de paramètres-laser utilisées. Notons enfin que l'amplitude de la modulation en puissance du laser est également relevée, de manière à estimer l'évolution de la réponse en fréquence dans le cas où le

Fig. 2. - Diagrammes indiquant les différents comportements dynamiques des lasers semiconducteurs suivant le taux et la fréquence de la modulation en courant, le taux de polarisation étant fixé à 1,5. La définition des comportemènts I, II, III, IV est donnée dans le texte. Les diagrammes $2 \mathrm{a}, \mathrm{b}, \mathrm{c}$ correspondent respectivement aux lasers I, II, III du tableau I. La droite horizontale en traits hachurés indique dans chaque diagramme la position de la fréquence de résonance $f_{\mathrm{R}}$ calculée en faible signal. Les triangles rapportés dans les diagrammes $2 \mathrm{a}, \mathrm{b}, \mathrm{c}$ indiquent les valeurs des paramètres de modulation pour lesquelles des évolutions temporelles sont présentées dans la figure 3.

[Diagrams indicating the various dynamic behaviours of semiconductor lasers according to the frequency - and rate - of current modulations. The normalized bias level is 1.5. The different types of behaviours (I, II, III, IV, respectively) are defined in the text. The diagrams $2 a, 2 b$ and $2 c$ correspond to lasers I, II and III of table I, respectively. In each diagram, the horizontal dashed line indicates the location of the resonance frequency, $f_{R}$, calculated in the small signal approximation. The black triangles reported in diagrams $2 \mathrm{a}, \mathrm{b}, \mathrm{c}$ indicate the modulation parameters values for which time-evolutions have been calculated in figure 3 .] comportement est le comportement périodique simple.

Les résultats obtenus pour un taux de polarisation égal à $\sim 1,5$ sont rapportés dans les figures $2 \mathrm{a}, \mathrm{b}, \mathrm{c}$. Les diagrammes de bifurcation correspondent respectivement aux trois lasers envisagés dans le tableau I. La ligne horizontale hachurée indique dans chaque cas la position de la fréquence de résonance en faible signal $f_{\mathrm{R}}$.

De manière générale quatre types de comportement ont été observés :

Comportement de type I (comportement périodique simple). - Ce comportement est celui souhaité.
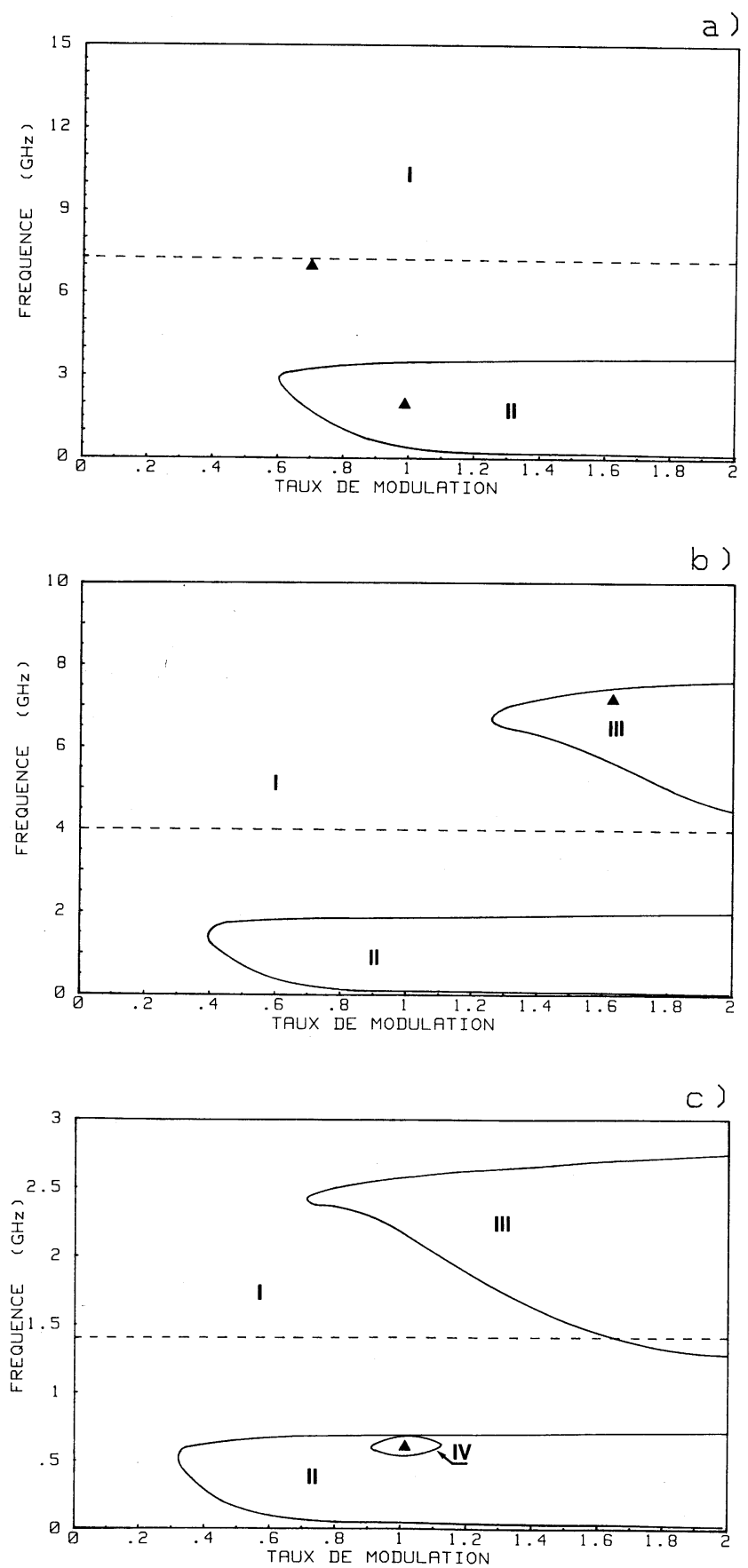
Contrairement à ce que suggéraient les travaux rapportés dans la référence [15], il correspond heureusement au domaine le plus étendu, quel que soit le laser envisagé. C'est dans le cas du laser InGaAsP à plus fort amortissement que l'extension du domaine est la plus grande. Notons en contrepartie que des effets de distorsion du signal optique se manifestent nettement dès que le taux de modulation dépasse $60 \%$, et ceci quelle que soit la fréquence de modulation. L'évolution temporelle rapportée dans la figure $3 \mathrm{a}$ en est une illustration.

Comportement de type II (comportement périodique simple avec présence de pics multiples par période de modulation). - Ce comportement est obtenu aux basses fréquences et pour des taux de modulation forts ou modérés. Dans le cas du laser InGaAsP présentant le plus fort amortissement, le taux de modulation-limite est d'environ $60 \%$. La figure $3 b$

a )

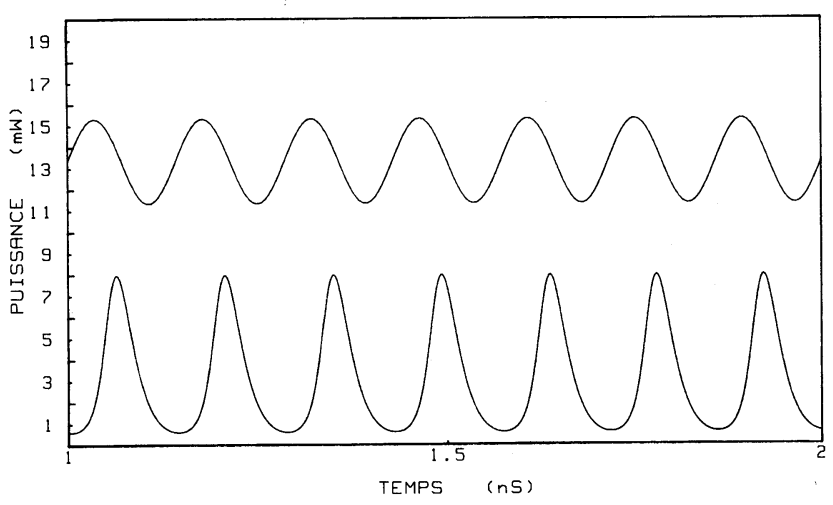

b)

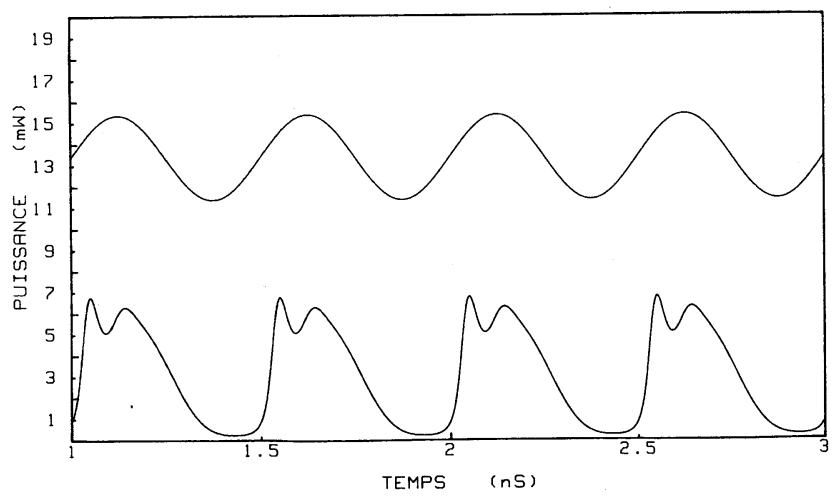

Fig. 3. - Evolutions temporelles du signal optique correspondant à différents types de comportement et/ou à différents lasers (courbes du bas). a) Comportement de type I - Laser I - taux de modulation $=0,77 ; b$ ) comportement de type II - Laser I - taux de modulation = 1 ; c) comportement de type III - Laser II - taux de modulation $=1,7$; d) comportement de type IV - Laser III - taux de modulation $=1$. Dans chaque cas la puissance optique est rapportée en vraie grandeur. La sinusoïde de la modulation en courant est aussi représentée (courbes en haut). Son amplitude et sa position verticale sont arbitraires. donne un exemple de comportement avec pics multiples pour ce laser. Le nombre de pics est ici limité à deux, mais peut être plus important dans le cas des lasers AsGa. La séparation entre pics est approximativement la période de résonance $\left(T_{\mathrm{R}}=1 / f_{\mathrm{R}}\right)$. L'application de pics multiples n'est donc qu'une manifestation des oscillations de relaxation du laser, celles-ci étant néanmoins altérées par la forme sinusoïdale de la modulation. Notons enfin que le comportement de type II a été obtenu pour toutes les valeurs de taux de polarisation envisagées et qu'il a été effectivement obșervé dans une étude récente de laser DFB à 1,3 $\mu \mathrm{m}$ [29].

Comportement de type III (doublement de période à haute fréquence de modulation). - Ce comportement est obtenu pour les deux lasers présentant les plus faibles amortissements. Il se produit à une fréquence légèrement inférieure à $2 f_{\mathrm{R}}$ et pour des

c )

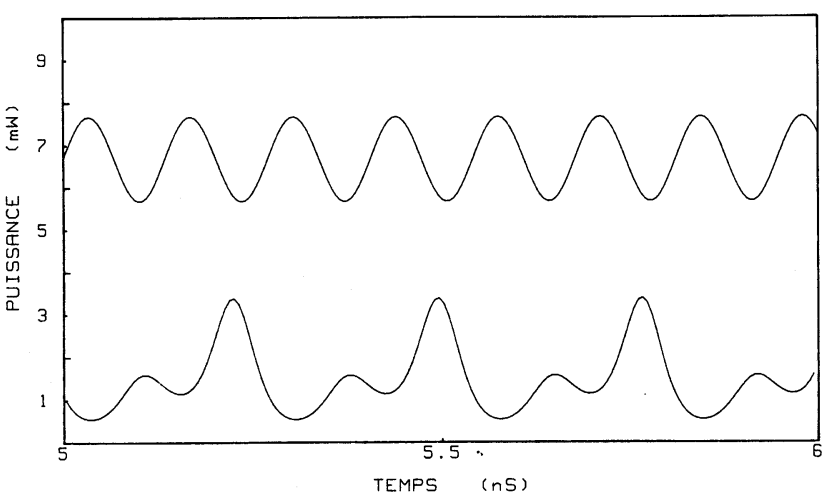

d)

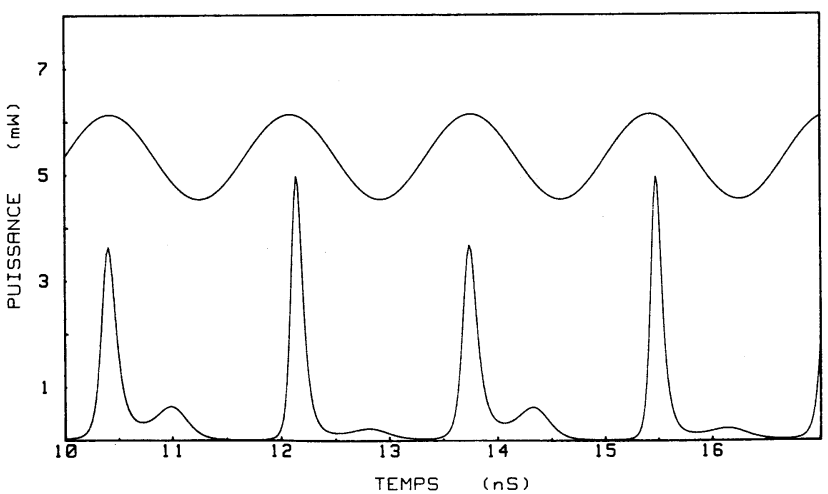

[Different time-evolutions of the optical signal corresponding to different types of behaviours and/or to different lasers (bottom curves). a) Behaviour of type I - Laser I modulation rate $=0.7$; b) behaviour of type II - Laser I modulation rate $=1 ; \mathrm{c}$ ) behaviour of type III - Laser II modulation rate $=1.7$; d) behaviour of type IV - Laser III - modulation rate $=1$. In each case, the optical signal is given in real unit. The sinusoidal modulation of the current is also presented (top curves). Its amplitude and vertical positions are arbitrary.] 
taux de modulation au moins de l'ordre de $70 \%$. La figure $3 c$ donne une illustration du comportement de type III dans le cas du laser AsGa le plus performant et pour un taux de modulation de 1,7. Notons que ce comportement disparaît pour les taux de polarisation faibles $(\leqslant 0,7)$. Ce résultat peut s'interpréter aisément à partir de l'évolution du facteur d'amortissement en fonction du taux de polarisation (voir Fig. $1 \mathrm{a}$ et $1 \mathrm{~b})$.

Comportement de type IV (doublement de période à basse fréquence de modulation). - Ce comportement n'est obtenu que pour le laser AsGa présentant le plus faible amortissement (diagramme 2c). A la différence du comportement de type III, il ne se produit que dans un domaine fermé de l'espace $3 \mathrm{~d}$ des paramètres de modulation. La fréquence d'apparition est proche de $f_{\mathrm{R}} / 2$, mais néanmoins légèrement inférieure. Les taux de modulation sont voisins de l'unité et les taux de polarisation compris entre $\sim 1,2$ et 1,8 . Une illustration de ce comportement est donnée dans la figure $3 d$. Le fait qu'il se produise au voisinage d'un sous-harmonique de la fréquence de résonance se comprend aisément par analogie avec l'oscillateur paramétrique [13b]. De même, l'existence de bornes inférieure et supérieure pour le taux de polarisation s'explique à partir de l'évolution du facteur d'amortissement avec ce paramètre. En revanche, le fait que le taux de modulation se situe nécessairement dans un intervalle limité, ne reçoit pas d'explication simple.

Une analyse plus fine du domaine IV du diagramme $2 c$ a été menée en prenant des pas de fréquence et de taux de modulation plus resserrés. Aucune bifurcation supplémentaire n'a été détectée qui aurait indiqué par là même la présence d'un domaine chaotique, même restreint. La prise en compte d'un terme de gain non linéaire $\varepsilon$ dans les équations-laser conduit donc à la suppression du régime chaotique. Ceci est en accord avec les résultats de la référence [16]. Des valeurs de $\varepsilon$ dix fois plus faibles ont cependant été envisagées ici, ce qui généralise le résultat à l'ensemble des dispositifs.

En revanche, la possibilité d'un doublement de période 'à basse fréquence de modulation pour les lasers AsGa n'est pas exclue. Ceci confirmerait ainsi les observations expérimentales de Y. C. Chen et al. [14], d'autant que les valeurs des paramètres de modulation pour lesquelles ce comportement a été observé sont proches de celles indiquées dans le diagramme $2 c$.

\subsection{PRÉDICTIONS DES COMPORTEMENTS DYNAMI- QUES À PARTIR DES COURBES DE RÉPONSE EN FAI- BLE SIGNAL.}

Comportement de type II (pics multiples). - Si l'on admet que la séparation entre deux oscillations de relaxation est simplement $T_{\mathrm{R}}=1 / f_{\mathrm{R}}$, alors la condi- tion pour observer au moins deux pics pendant une période de modulation s'écrit :

$$
f<f_{\mathrm{R}} / 2 \text {. }
$$

Cette condition est tout à fait vérifiée sur les diagrammes $2 a, b, c$. Il est d'autre part évident que l'observation effective de pics multiples implique une dynamique importante (fort taux de modulation) et une valeur pas trop élevée du coefficient d'amortissement.

Comportements de types III et IV (doublement de période). - L'existence de comportements instables tels que ceux de types III et IV ne peut être prédite précisément, étant donné la nature non linéaire du problème. Nous admettrons ici aussi que ces comportements sont liés aux oscillations de relaxation du laser et que deux conditions sont nécessaires :

i) Le temps d'amortissement des oscillations de relaxation $\left(1 / \xi \omega_{0}\right.$, voir paragraphe IIIb) est supérieur à la période de modulation $(1 / f)$. En d'autres termes, des phénomènes d'interférence se produisent sur deux périodes consécutives. Cette condition est identique à celle énoncée par Agrawal [16].

ii) Les instabilités ne peuvent se développer que si la fréquence de modulation est un harmonique ou un sous-harmonique d'ordre pair de la fréquence de résonance ( $f \approx n \times f_{\mathrm{R}}$ ou $f_{\mathrm{R}} / n$ avec $n$ pair) et l'on ne considérera ici que la valeur $n=2$. En réalité, les résultats de simulation numérique montrent que $f$ est toujours sensiblement inférieure aux valeurs indiquées précédemment. Tout se passe comme si, en fort signal, la fréquence de résonance était déplacée vers les plus faibles valeurs [2]. Le relevé de la valeur numérique de l'amplitude de la modulation en puissance du laser nous a permis de vérifier cette assertion.

Si l'on suppose en outre que la fréquence de résonance $f_{\mathrm{R}}$ est proche de la fréquence propre $f_{0}$ alors les conditions i) et ii) se ramènent à une condition simple sur le facteur d'amortissement.

Pour qu'un comportement de type III puisse apparaître, il faut :

$$
1 / 2 \xi>\pi / 2
$$

soit une amplitude à la résonance d'au moins 4,5 dB pour la réponse en faible signal.

Pour qu'un comportement de type IV puisse apparaître, il faut :

$$
1 / 2 \xi>2 \pi
$$

soit une amplitude à la résonance d'au moins $16 \mathrm{~dB}$.

Les conditions (11a) et (11b) fournissent une interprétation tout à fait satisfaisante des résultats de simulation numérique. L'examen des courbes de réponse en faible signal (Fig. 1a et 1b) montre en 
effet qu'un comportement de type IV ne peut en aucun cas se produire pour les deux lasers considérés (lasers «performants » InGaAsP et AsGa). La comparaison des conditions (11a) et (11b) indique par ailleurs que le doublement de période à haute fréquence de modulation est plus facilement observable que celui à basse fréquence. Le comportement de type III est en effet obtenu pour les deux lasers AsGa considérés dans la simulation numérique ; il est à la limite d'être obtenu pour le laser InGaAsP. D'autres tests numériques ont été également effectués pour d'autres valeurs du terme de gain non linéaire $\varepsilon$, confirmant la validité des conditions (11a) et $(11 \mathrm{~b})$, bien qu'étant approchées.

Comportement chaotique. - Si l'on admet que le domaine chaotique est inclus dans le domaine IV et seulement dans ce domaine, alors la condition d'apparition du chaos est encore plus restrictive que la condition (11b). L'hypothèse précédente (domaine chaotique inclus dans le domaine IV) a été vérifiée dans le cas où $\varepsilon=0$ [15]. Nous l'avons également vérifiée pour des valeurs de $\varepsilon$ très faibles, mais différentes de zéro. La conclusion à laquelle nous aboutissons concernant le régime chaotique diffère donc fondamentalement de celle avancée par Agrawal [16]. Cet auteur suggérait en effet que le chaos pouvait réapparaître à haute fréquence de modulation $(f \geqslant 40 \mathrm{GHz})$ et donc pour des lasers susceptibles de répondre à une modulation rapide. Or, ceci revient à supposer, soit que le chaos se produise à une fréquence harmonique de la fréquence de résonance (ex. : domaine III), ce qui n'est manifestement pas le cas, soit que des lasers à modulation rapide présentent forcément de très faibles amortissements, ce qui n'est pas non plus vérifié dans les exemples actuels $[8,11]$.

\section{Conclusion.}

Une étude détaillée des comportements des lasers à semiconducteurs modulés en courant vient d'être présentée lorsque des taux de modulation élevés sont mis en jeu.

Il est démontré que l'existence de tel ou tel comportement est essentiellement liée au facteur d'amortissement du laser et donc qu'elle peut être diagnostiquée à partir des courbes de réponse en faible signal.

En ce qui concerne les lasers InGaAsP à 1,3 $\mu \mathrm{m}$ dont l'amortissement est en général élevé, il apparaît que les comportements de types chaotique ou « doublement de période » ne puissent être observés. Ceci est en accord avec l'étude récente de G. P. Agrawal [16]. En revanche, les résultats de simula- tion numérique montrent que, même dans le cas du comportement périodique simple, une distorsion importante du signal optique doit être observée pour des taux de modulation supérieurs à $70 \%$ et qu'à basse fréquence de modulation des oscillations de relaxation doivent être détectées à l'intérieur de chaque période de modulation. Expérimentalement, les effets de distorsion se traduiront à l'évidence par un spectre de puissance riche en harmoniques. Cependant, dans ce cas, une confrontation précise théorie-expérience nécessite d'utiliser un modèle en champ et non plus un modèle en intensité comme celui qui est présenté ici. Ceci ne pose a priori aucune difficulté de principe dans la mesure où le laser reste effectivement monomode [30]. D'un autre côté, la détection expérimentale de pics multiples par période de modulation peut être faite par un échantillonnage direct du signal optique. Seuls les effets associés au bruit d'émission spontanée du laser (« jitter » d'une impulsion optique à l'autre...) et qui ne sont pas pris en compte dans le présent modèle peuvent éventuellement rendre la détection difficile.

En ce qui concerne les lasers AsGa dont l'amortissement est plus faible, les résultats de simulation numérique montrent que des doublements de période à basse ou à forte fréquence de modulation peuvent se produire en plus des comportements rappelés ci-dessus. En revanche, la possibilité d'un comportement chaotique semble aussi devoir être exclue. Expérimentalement, la détection de sousharmonique dans le spectre de puissance du laser ne doit pas poser de difficultés majeures. Le doublement de période à basse fréquence de modulation semble avoir été effectivement détecté [14]. Celui à haute fréquence de modulation reste à démontrer.

De manière plus générale, l'intérêt des lasers semiconducteurs modulés en courant s'étend maintenant aux dispositifs à puits quantiques multiples, dont les performances en fréquence devraient être largement supérieures à celles des lasers à double hétérostructure. L'étude qui vient d'être développée mérite donc d'être étendue à ces nouveaux dispositifs. Bien qu'elle fournisse déjà des indications de base, son extension passe par l'établissement d'un modèle suffisamment précis et généralisable à l'ensemble des dispositifs à puits quantiques multiples.

\section{Remerciements.}

Nous tenons à remercier P. Glorieux du Laboratoire de Spectroscopie Hertzienne (UA n ${ }^{\circ} 249$ du CNRS) dont l'expérience en matière $d^{\prime}$ ' instabilités-laser » nous a été particulièrement précieuse au départ de cette étude. 


\section{Bibliographie}

[1] Suematsu, Y., Proc. IEEE 71 (1983) 692.

[2] LAU, K. Y., YARIV, A., IEEE J. Quantum Electron. QE-21 (1985) 121.

[3] TAYlor, A. J., TuCKer, R. S., Wiesenfeld, J.-M., Burrus, C. A., Eisenstein, G., TAlman, J. R., PEI, S. S., Electron. Lett. 22 (1986) 1068.

[4] LAU, K. Y., Bar Chaim, N., URY, I., HaRder, Ch., Yariv, A., Appl. Phys. Lett. 43 (1983) 1.

[5] TUCKER, R. S., POPE, D. J., IEEE Trans. Microwave Theory Tech. MTT-31 (1983) 289.

[6] TUCKER, R. S., KAMINOw, J., J. Lightwave Technol. LT-2 (1984) 385.

[7] LaU, K. Y., Harder, Ch., Yariv, A., Appl. Phys. Lett. 44 (1984) 273.

[8] LaU, K. Y., Bar Chaim, N., URY, I., YARIv, A., Appl. Phys. Lett. 45 (1984) 316.

[9] TuCKer, R. S., J. Lightwave Technol. LT-3 (1985) 1180.

[10] Su, C. B., LANZisera, V., Olshansky, R., PowazINIK, W., MEland, E., SCHLAFER, J., LAUER, R. B., Electron. Lett. 21 (1985) 577.

[11] Olshansky, R., Lanzisera, V., Su, C. B., PowazINIK, W., Lauer, R. B., Appl. Phys. Lett. 49 (1986) 128.

[12] Krakowski, M., Blondeau, R., Kazmierski, K., RAzeghi, M., Ricciardi, J., HIRTZ, P., DE Cremoux, B., J. Lightwave Technol. LT-4 (1986) 1470.

[13] a) Berge, P., Pommeau, Y., Vidal, Ch., L'ordre dans le chaos Hermann Ed. (1984) p. 107.

b) Idem, p. 30-45.

[14] Chen, Y. C., Winful, H. G., LiU, J. M., Appl. Phys. Lett. 47 (1985) 208.

[15] Tang, M., Wang, S., Appl. Phys. Lett. 48 (1986) 900.
[16] Agrawal, G. P., Appl. Phys. Lett. 49 (1986) 1013.

[17] AsAda, M., Suematsu, Y., IEEE J. Quantum Electron. QE-21 (1985) 434.

[18] Olshansky, R., Su, C. B., Manning, J., PowazINIK, W., IEEE J. Quantum Electron. QE-20 (1984) 838.

[19] Mozer, A. P., Hausser, S., Pilkuhn, M. H., IEEE J. Quantum Electron. QE-21 (1985) 719.

[20] Sugimura, A., Appl. Phys. Lett. 39 (1981) 21.

[21] Furuya, K., Suematsu, Y., Hong, T., Appl. Opt. 17 (1978) 1949.

[22] TUCKer, R. S., POPE, D. J., IEEE J. Quantum Electron. QE-19 (1983) 1179.

[23] Leclerc, D., Brosson, P., Fernier, B., Benoit, J., IEE Proc. Part J 132 (1985) 28.

[24] Brosson, P., Fernier, B., Leclerc, D., Benoit, J., IEEE J. Quantum Electron. QE-21 (1985) 179.

[25] Pidgeon, C. R., Wherrett, B. S., Johnston, A. M., Dempsey, J., Miller, A., Phys. Rev. Lett. 42 (1979) 1785.

[26] Bowers, J. E., Koch, T. L., HeMENWAy, B. R., Wilt, D. P., BRIDGES, T. J., BURKHARDT, E. G., Electron. Lett. 21 (1985) 297.

[27] Paraskevopoulos, A., Devoldere, P., Hemery, E., Bouley, J. C. (communication privée).

[28] Gear, W., Numerical initial value problems in ordinary differential equations (Prentice Hall) 1971, p. 85.

[29] Yoshikuni, Y., Matsuoka, T., Motosugi, G., Yamanaka, N., Appl. Phys. Lett. 45 (1984) 820.

[30] Agrawal, G. P., IEEE J. Quantum Electron. QE21 (1985) 680. 